\title{
VENTROMEDIAL NUCLEUS OF HYPOTHALAMUS IS RELATED TO THE DEVELOPMENT OF CANCER-INDUCED ANOREXIA: IN VIVO MICRODIALYSIS STUDY
}

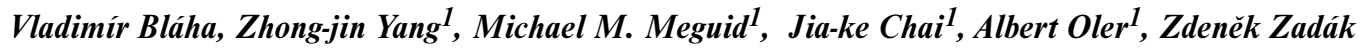 \\ Department of Metabolic Care and Gerontology, Charles University, Faculty of Medicine and Teaching Hospital, \\ Hradec Králové; (Head: prof. MUDr. Z. Zadák, CSc.) \\ Neuroscience Program, Surgical Metabolism and Nutrition Laboratory, Departments of Surgery and Pathology, \\ University Hospital, SUNY Health Science Center, 750 E. Adams Street, Syracuse, NY 132101; \\ (Head: M. M. Meguid, M.D., Ph.D., FACS)
}

\begin{abstract}
Summary: Based on reports that increased hypothalamic ventromedial nucleus (VMN) - serotonin (5-HT) is associated with cancer anorexia and recent findings in our laboratory that low levels of dopamine (DA) in the VMN are associated with prolonged inter meal intervals thus decreased food intake, and reports that setting up satiation is concomitant with descending levels of DA in the rostromedial hypothalamus, we hypothesized that an elevated 5-HT to low DA ratio in the VMN modulates food intake in cancer anorexia. Methods: In Expt 1: A microdialysis cannula guide was placed stereotactically into the VMN of methylcholanthrene (MCA) sarcoma tumor-bearing (TB) Fischer rats and in non-tumor-bearing (NTB) and pair-fed (PF) controls. When TB rats manifested anorexia by a decrease in food intake, VMN-5-HT, its metabolite 5-hydroxyindolacetic acid (5-HIAA), and DA with its metabolite 3,4,-dihydroxyphenylacetic acid (DOPAC) were measured by in vivo microdialysis using HPLC during baseline, in response to food, and after feeding. In Expt 2: TB rats had tumor removed and VMN microdialysis performed 7 days later. Results: Increased 5-HT release and turnover, and significantly reduced DA release with increased DOPAC occured in TB vs NTB or PF rats. When food was offered, intake in TB rats was significantly lower than in NTB control rats. During eating, VMN-5-HT rose and peaked significantly earlier in TB vs NTB rats, while DA release was significantly reduced. With eating, the 5-HT and DA metabolism became reduced in all rats. Seven days after surgical removal of the tumor, $24 \mathrm{~h}$ food intake had increased to the level of controls; and when food was offered during microdialysis, intake in TB rats increased (ns relative to control), but was not yet normal. VMN microdialysis showed that 5-HT was normal at baseline, as well as during and after eating, while DA remained depressed. The metabolic turnover of 5-HT and DA was significantly lower in TB-r and PF vs NTB rats. We conclude that increased 5-HT/DA ratio is related to the development of cancer-induced anorexia.
\end{abstract}

Key words: Food intake; Ventromedial nucleus of hypothalamus (VMN); Neurotransmitters; Serotonin (5-HT); Dopamine (DA); 5-hydroxyindolacetic acid (5-HIAA); 3,4,-dihydroxyphenylacetic acid (DOPAC); Cancer anorexia

Work was supported in part by grant DK43796 from the National Institutes of Diabetes and Digestive and Kidney Diseases, NIH; by grant 85A95 from the American Institute for Cancer Research; and by grant IGA MZ ČR 4095-3.

\section{Introduction}

Anorexia is a major initiating factor in the development of the cachectic state in cancer. Considering anorexia as loss of a desire to eat, several candidates are frequently cited as putative anorectic factors in cancer anorexia (for review see 25): cytokines (27), products of glucose, fat and amino acid metabolism (9), and more recently, neuropeptide Y (11), as well as other hypothalamic neurotransmitters $(7,15,16,18,20,23,25)$.
Early satiety, as one characteristic, of cancer anorexia has been often associated with elevated brain serotonin (5HT) levels and metabolism $(5,7,9,22,24,37)$. Analyzing the involvement of brain neurotransmitters in vitro, the phenomenon of cancer anorexia involved an increase of 5-HT, and, moreover, its precursor tryptophan (TRP) and its metabolite 5-hydroxyindolacetic acid (5-HIAA) $(5,7,9,37)$ in MCA-induced and/or Walker 256 carcinosarcoma-induced anorexia. The brain levels of TRP, 5-HT and 5-HIAA were not elevated in the pair-fed animals vs TB rats. Therefore it 
appeared that the elevated serotonergic activity did not continue to increase in direct proportion to the degree of malnutrition (5), suggesting that the brain 5-HT may be involved in the development of cancer anorexia.

The hypothalamus is a primary mediation site of monoamine and neuropeptide effects on food intake (16); when studying rat models of cancer anorexia in vitro, regional analysis of elevated serotonergic activity was observed in several brain areas including hypothalamus and several extra-hypothalamic sites $(5,7,8,22,24,37)$. Hypothalamic serotonergic system-mediated cancer anorexia may be localized into the VMN. In anorectic tumor-bearing rats, when bilateral intra-VMN colchicine is given, anorectic tumor-bearing rats restore their food intake to normal, and $60 \%$ increase in food intake in non tumor-bearing rats occurs (20). Food intake was increased for two days after selective intra-VMN mianserin (5-HT antagonist [1]) injection in anorectic tumor-bearing rats, while no effect was observed from systemic or intracysternal 5-HT depletion (6). Furthermore, the concentration of cerebrospinal IL-1a correlated with anorexia and tumor weight in MCA-bearing rats (27). This central effect of IL1 seems to be 5-HT mediated, because IL-1b induced activation of the serotoninergic system was documented in medial basal hypothalamus ( $\mathrm{MBH}$ [22]). We have also shown that anorectic tumor-bearing rats injected into the VMN with a selective IL-1 receptor antagonist (IL-1 ra) (13) increased their food intake. Based on these data, serotonergic-VMN impairment during tumor growth appears to be an essential step that leads to a decrease in food intake (20).

Dopamine (DA) has been shown to be either stimulatory or inhibitory on feeding. When inhibitory, its action was repeatedly reported to be localized in the lateral hypothalamic area (LHA) $(4,14,6,31)$. Although an increase in hypothalamic DA release has been reported in tumor anorexia (8), the anorexigenic effect of IL-1a was not mediated through dopaminergic activity in the LHA (38). The feeding behavioral role of DA in satiety-related centers as VMN and PVN is not clear. In the normal rat, post-prandial satiety is associated with eating-induced low VMN DA (21). Decreasing levels of VMN-PVN DA were reported to cause satiation and stop feeding (28). Until recently, most of the approaches to measure brain DA activity in the tumor-bearing animals used in vitro methods. Thus, significant differences in the whole hypothalamus DA $(7,9)$ or both its metabolite 3,4,-dihydroxyphenylacetic acid (DOPAC) (8) were not reported in anorectic tumor-bearing rats, while a significant increase of DA and its metabolite DOPAC had been shown in anorectic Morris hepatoma-bearing male Buffalo rats (8).

The introduction of the technique of in vivo brain microdialysis in the awake animal allows the concomitant measurement of behavior and neurochemical correlates, and solves some problems of in vitro brain measurements. Therefore, based on the previous established reports of inc- reased VMN-5-HT/5-HIAA associated with cancer anorexia and recent reports that low levels of DA in the VMN are associated with post-prandial satiety, we hypothesized that high VMN-serotonergic and low VMN-dopaminergic activities may modulate food intake in cancer anorexia. This hypothesis was tested in anorectic MCA-bearing rats using the in vivo microdialysis technique.

\section{Material and methods}

\section{Subjects:}

The experiment was approved by the State University of New York Health Science Center, Syracuse, Committee for the Humane Use of Animals, and was in accordance with the guidelines established by the National Institutes of Health. Fischer 344 male rats were housed in holding wire cages for 10 days after purchase to acclimatize them to the constant study enviromental conditions: 12-h light-dark cycle (lights on at 0600$), 26 \pm 1^{\circ} \mathrm{C}$ room temperature, and $45 \%$ humidity.

After 10-day acclimatization period, rats were randomly assigned to the three experimental groups: non-tumor-bearing (NTB, $n=9)$; pair-fed non-tumor-bearing ( $P F, n=5)$; and tumor-bearing $(\mathrm{TB}, \mathrm{n}=6)$ rats.

All rats were allowed free access to water and standard rat chow (diet 5008, Ralston Purina, St. Louis, MO). Before microdialysis, the NTB group was fasted overnight. The PF animals were given food as indicated. Body weights were measured gravimetrically every 3 days. Daily food intakes were recorded.

\section{Tumor Model:}

Controls and pair-fed rats received a subcutaneous inoculation of $1 \mathrm{ml}$ normal saline into the right flank, while tumor-bearing rats were given a $1 \mathrm{ml}$ inoculum containing $10^{6}$ methylcholanthrene (MCA) -induced sarcoma cells, prepared according to the method of Madden and Burk (17), from a MCA tumor freshly excised from the donor. This tumor characteristically grows locally and induces fatality 35 45 days after implantation.

The injection site in the right flank was palpated daily to detect growth of the injected tumor cells; when palpable, the length and width of the tumor were measured with calipers. Tumor weight was calculated from the formula for a prolate spheroid, $V=1 / 2 a b^{2}$, where $a$ is the longer, $b$ is the shorter dimension (12) and tumor density taken as 1.0. The calculated tumor weight using this formula correlated with the actual weight of freshly excised tumors in our present study. Cancer anorexia was defined as the third day in which food intake was less than $1 \mathrm{~g} / 100$ body weight than the mean food intake of the controls (7).

To estimate nontumor body weights of the TB group, the carcass body weight was calculated by subtracting estimated tumor weights from the total body weights of the TB rats: The results are shown in Table 1 (TB-carcass group). 
Table 1: Body weight (g) throughout the experiment. Rats were assigned to TB, NTB or PF groups from the day 0 , when the tumor was removed in the TB group. Body weights were measured gravimetrically every 3 days. The results are shown as mean $\pm \mathrm{SE}$ for carcass weight of tumor-bearing (TB-carcass), non-tumor-bearing (NTB) and non-tumor-bearing pair-fed (PF) rats.

\begin{tabular}{|c|c|c|c|}
\hline Days from tumor removal & TB-carcass & NTB & PF \\
\hline-15 & $287 \pm 4$ & $291 \pm 5$ & $292 \pm 5$ \\
-12 & $287 \pm 4$ & $295 \pm 5$ & $299 \pm 3$ \\
-9 & $285 \pm 5$ & $297 \pm 5$ & $303 \pm 2$ \\
-6 & $281 \pm 9$ & $296 \pm 4$ & $302 \pm 4$ \\
-3 & $257 \pm 13$ & $301 \pm 5$ & $297 \pm 3$ \\
0 & $242 \pm 7$ & $295 \pm 6$ & $282 \pm 6$ \\
+3 & $232 \pm 4$ & $310 \pm 1$ & $274 \pm 5$ \\
+6 & $236 \pm 6$ & $314 \pm 1$ & $277 \pm 3$ \\
\hline
\end{tabular}

\section{Pair Feeding:}

A group of non-tumor-bearing control animals (PF) was used to control for the possible effects of food deprivation on the hypothalamic neurotransmitters. Individual PF rats were offered the amount of food/24 hr that was consumed by rats from the TB group, both while bearing tumor and after tumor removal.

In our pilot study, when food was offered to the food-deprived non tumor-bearing PF animals during the microdialysis period, the average size of the first meal ranged between 5-8 g. Therefore, to mimic exactly the feeding pattern of TB rats, PF animals were offered the same reduced amount of food also during the microdialysis period.

\section{Ventromedial Hypothalamus Cannulation:}

Four days after MCA cells or saline injection, rats were anesthetized with a mixture of Ketamine, Xylazine and Acepromazine, $150: 30: 5 \mathrm{mg} / \mathrm{ml}$, at $0.5-0.7 \mathrm{ml} / \mathrm{kg}$ body weight intramuscularly. The rat was placed in the stereotaxic instrument and the skull exposed on top of the head. An intracerebral cannula guide to the VMN was implanted at stereotaxic coordinates from the bregma: medio-lateral, 1.0 $\mathrm{mm}$ from the middle line; and dorsal-ventral, $8.6 \mathrm{~mm}$ ventral from the surface of the dura (32). The cannula guide was fixed to the skull with acrylic dental cement. After operation, rats were kept individually in the plastic metabolic cages (Nalgene Company, Rochester, NY) and were allowed to recover for 10 days.

\section{Experimental Design:}

When the TB rats became anorectic, the first microdialysis was performed. Immediately after microdialysis, on the same day, tumors were surgically resected under enflurane (Ethrane ${ }^{\circledR}$ ) anesthesia using aseptic techniques. Seven days later, when the $24 \mathrm{hr}$ food intake had returned to control levels, the second microdialysis was performed (TB-r).
The control groups of NTB and PF rats were microdialysed for the first time $20 \pm 2 \mathrm{~d}$ after vehicle injection. Immediately after microdialysis, on the same day, they were subjected to a sham operation which involved incising and suturing of dorsal skin under enflurane $\left(\right.$ Ethrane $\left.^{\circledR}\right)$ anesthesia using aseptic surgical techniques. Seven days later, the second microdialysis was performed (Fig. 1).

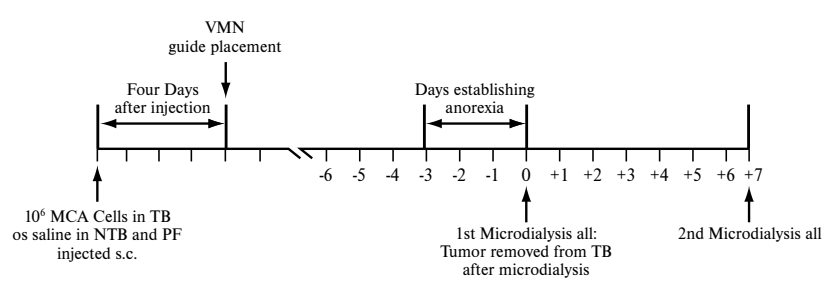

Fig. 1: Experimental protocol. After 10-day acclimatization period, rats were randomly assigned to the three experimental groups: non-tumor-bearing $(\mathrm{NTB}, \mathrm{n}=9)$; pair-fed non-tumor-bearing $(\mathrm{PF}, \mathrm{n}=5)$; and tumor-bearing $(\mathrm{TB}, \mathrm{n}=6)$ rats. Controls and pair-fed rats received a subcutaneous inoculation of $1 \mathrm{ml}$ normal saline into the right flank, while tumor-bearing rats were given a $1 \mathrm{ml}$ inoculum containing $10^{6}$ methylcholanthrene (MCA) -induced sarcoma cells. Four days after injection, a guide cannula was placed to the VMN. Anorexia was defined as food intake $<1 \mathrm{~g}$ food $/ 100 \mathrm{~g}$ body weight consumed for 3 consecutive days, i.e., days $-3,-2$, and -1 . Thus day 0 is the first day after anorexia is established. When the TB rats became anorectic, the first microdialysis was performed. Immediately after microdialysis, on the same day, tumors were surgically resected. Seven days later, when the 24 $\mathrm{hr}$ food intake had returned to control levels, the second microdialysis was performed (TB-r). The control groups of NTB and $\mathrm{PF}$ rats were microdialysed for the first time $20 \pm 2 \mathrm{~d}$ after vehicle injection. Immediately after microdialysis, on the same day, they were subjected to a sham operation. Seven days later, the second microdialysis was performed.

\section{Microdialysis Design:}

At 8:00 AM, the anorexic TB, TB-r, PF or overnight fasted non-anorexic NTB rat was put into a bowl-like cage (CMA/120 Awake Animal System, BAS, West Lafayette, IN). A microdialysis probe was inserted into the guide cannula, extending $1 \mathrm{~mm}$ below the cannula into the $\mathrm{VMH}$ at 9:00 AM. After a 60 minute stabilization period, three 20 minute baseline dialysates were collected and monoamine levels measured to make sure that baseline levels were consistent. Until this time, the rats had no access to food. After the three $20 \mathrm{~min}$ dialyses while in the microdialysis cage, food pellets were provided ad libitum to the TB, TB-r and NTB groups; PF rats were offered the same amount of food as the TB and/or TB-r animals had eaten. VMN dialysates were continuously collected every 20 minutes for 2 hours while the rats had access to food. Simultaneously, the rat feeding activity was recorded. 


\section{Microdialysis Procedure:}

The CMA/10 microdialysis probe was used (BAS, West Lafayette, IN). The microdialysis membrane was $1 \mathrm{~mm}$ long, $400 \mathrm{~mm}$ ID, $520 \mathrm{~mm}$ OD., and 20,000 Dalton molecular weight cutoff. According to our in vitro calibration test, a relative recovery rate was about $10-12 \%$ for DA and 6 $8 \%$ for 5 -HT at a flow rate $1 \mathrm{ml} /$ minute. A Ringer type solution containing $147.0 \mathrm{mM} \mathrm{Na}^{+}, 2.4 \mathrm{mM} \mathrm{Ca}^{++}, 4.0 \mathrm{mM} \mathrm{K}^{+}$ and $155.8 \mathrm{~m} \mathrm{M} \mathrm{Cl}^{-}$was used to perfuse through the microdialysis membrane. The flow rate $1 \mathrm{ml} / \mathrm{min}$ allowed the collection of $20 \mathrm{ml}$ samples every 20 minutes into the microvials.

\section{Monoamines Measurement:}

All samples were measured immediately after collection. Monoamines were detected using a reverse phase liquid chromatography with ESA Model 5014 High Sensitivity Analytical Cell and ESA MD-150 column (3mm, 150mm $\mathrm{x} 3 \mathrm{~mm}$ ID). The mobile phase consist of $75 \mathrm{mM}$ $\mathrm{NaH} 2 \mathrm{PO} 4 . \mathrm{H} 2 \mathrm{O}, 1.4 \mathrm{mM}$ OSA, $10 \mathrm{mM}$ EDTA, and 10\% acetonitrile. Buffer $\mathrm{pH}$ is adjusted to 3.1 with $\mathrm{H} 3 \mathrm{PO} 4$. The concentration of monoamines was determined by comparison with peak areas of standards run with each experiment.

\section{Histology:}

The locations of the dialysis probe in the VMN were verified as previously described $(19,38,39)$ using the stereotaxic atlas of Pellegrino et al. (32).

\section{Data Analysis:}

The baseline concentrations of 5-HT, 5-HIAA, DA and DOPAC are expressed in pg per $10 \mathrm{ml}$ dialysate. It is important to indicate that these values are not the actual extracellular 5-HT, 5-HIAA, DA and DOPAC concentrations in the VMN because recovery procedures do not take into account diffusion kinetics in brain tissue. The baseline 5-HT, 5-HIAA DA and DOPAC levels were determined by using the mean value from 3 consecutive samples prior to eating. In the NTB group, the baseline monoamines were not different during the first and second microdialysis. Therefore, the data were pooled together and are presented as such.

Eating-related changes in 5-HT, 5-HIAA, DA and DOPAC are expressed as percent variations from the mean baseline values preceding the eating.

All data are expressed as mean \pm SE. ANOVA was used to analyze the time-course data within the groups while, the two-tailed Student's t-test was used to compare between NTB, PF and TB/TB-r rats.

\section{Results}

\section{Animal and Tumor weights}

When the animals became anorectic, according to the definition given above, this was taken as Day 0 . This varied days $19 \pm 2$ from tumor inoculation. Tumor became palpable in all rats 4 to 6 days after inoculation. The actual weight of freshly excised tumors as weighted on Day 0 was $80 \pm 8 \mathrm{~g}$.
At the start of experiment, when the rats were injected with tumor cells or vehicle, the body weights of the animals were not significantly different $(\mathrm{TB}=298 \pm 4 \mathrm{~g}, \mathrm{NTB}=294 \pm$ $5 \mathrm{~g}, \mathrm{PF}=299 \pm 4 \mathrm{~g})$. Although total body weight of the tumorbearing rats was increasing significantly $(p<0.05)$ from that of the non-tumor-bearing group from Day - 6 onward, much of this difference consisted of tumor weight. Subtraction of the estimated tumor weights from the total body weights of the tumor-bearing rats yielded carcass body weights (TB-carcass), as shown in Table 1. From Day -12, the mean carcass weight (TB-carcass) was significantly lower than NTB or PF control's body weight, and continued to decline until tumor removal on Day 0. PF rats began to lose weight after pair feeding began (Day -5), and showed both a significant attenuation of weight gain compared with NTB controls by Day 0 .

That the carcass weight loss of TB rats was greater than that of PF controls can be seen in Table 1. Although their daily food intakes were similar throughout the study period, the loss in body weight of TB rats from the Days -12 was significantly greater compared with the PF controls $(p<0.05)$ or ad libitum-fed NTB rats $(\mathrm{p}<0.05)$. The body weight of TB increased after tumor removal, but remained signicantly lower vs NTB or PF rats on Day $+6(\mathrm{p}<0.05)$.

\section{Effect of MCA Sarcoma on Food Intake}

Food Intake per $24 \mathrm{hr}$. Food intake of TB rats had decreased significantly compared with NTB controls by Day 6 and continued to decline until the rats had their tumor excised on Day 0 . On Day +6 , seven days after tumor removal, the $24 \mathrm{hr}$ food intake in the TB rats had increasedto that of the NTB group (Fig. 2). The pair-fed animals (PF) were offered the equal amount of food as the TB rats.

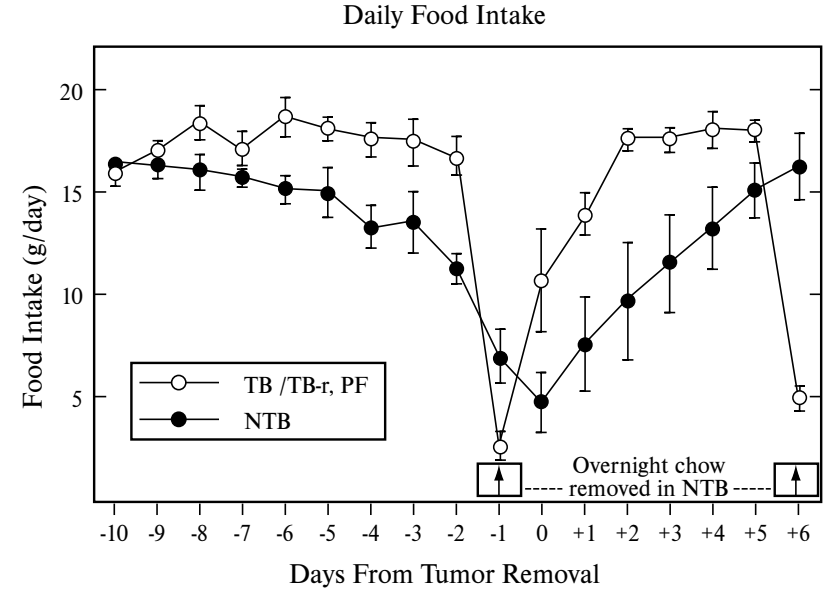

Fig. 2: Daily food intake (g/day). The results are shown as mean \pm SE for tumor-bearing (TB), TB after tumor removal (TB-r), non-tumor-bearing (NTB) and non-tumor-bearing pair-fed (PF) rats. Before microdialysis, the NTB group was fasted overnight, as indicated by arrow. Individual PF animals were offered the amount of food $/ 24 \mathrm{hr}$ that was consumed by rats from the TB group, both while bearing tumor and after tumor removal. 
Food Intake during Microdialysis. When food was offered during microdialysis, intake in TB vs NTB rats was significantly lower $(0.7 \pm 0.2 \mathrm{~g}$ vs $3.0 \pm 0.4 \mathrm{~g}, \mathrm{p}<0.01)$ (Fig. 4$)$. After tumor removal, food intake during microdialysis was still lower in TB-r than NTB rats $(1.2 \pm 0.4 \mathrm{~g}$ vs $3.0 \pm 0.4 \mathrm{~g}$, $\mathrm{p}<0.01$ ) (Fig. 3). The pair-fed animals (PF) were offered the equal amount of food as the TB rats and ate it all promptly.

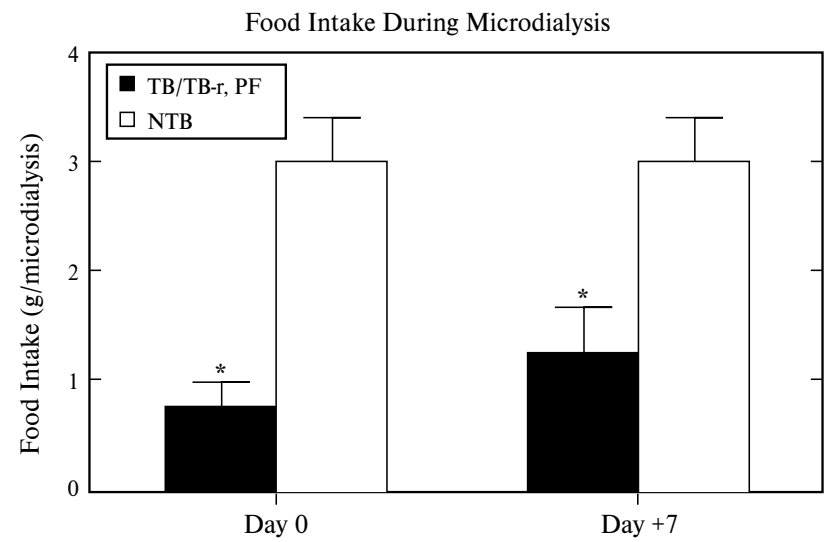

Fig. 3: Food intake during microdialysis. First microdialysis was done during anorexia on Day 0 (left); and second microdialysis was done seven days after tumor removal on Day +7 (right). To mimic exactly the feeding pattern of TB rats, PF animals were offered the same reduced amount of food also during the microdialysis period. The results are shown as mean \pm SE for tumor-bearing (TB), TB after tumor removal (TB-r), non-tumor-bearing (NTB) and non-tumor-bearing pair-fed (PF) rats. Significance * $\mathrm{p}<0.05$ vs NTB.

\section{Baseline 5-HT, 5-HIAA, DA and DOPAC.}

During anorexia on Day 0, as summarized in Table 2, baseline VMN-5-HT and 5-HIAA in TB were significantly higher vs PF $(\mathrm{p}<0.01)$ and overnight fasted NTB rats $(\mathrm{p}<$ $0.01)$. VMN 5-HIAA was significantly higher in TB ( $p<$ $0.01)$ and significantly lower in PF animals $(\mathrm{p}<0.01)$ compared to NTB control.

Tab. 2: Baseline VMN-5-HT, 5-HIAA, DA and DOPAC values $(\mathrm{pg} / 10 \mathrm{ml})$ during anorexia (Day 0) and seven days after the animals had tumor resected (Day +7$)$. The results are shown as mean \pm SE for tumor-bearing (TB), TB after tumor removal (TB-r), non-tumor-bearing (NTB) and nontumor-bearing pair-fed $(\mathrm{PF})$ rats. Significance ${ }^{*} \mathrm{p}<0.01$ vs $\mathrm{NTB},{ }^{+} \mathrm{p}<0.01$ vs PF or TB, ${ }^{0} \mathrm{p}<0.01$ Day 0 vs Day +7 .

\begin{tabular}{|l|c|c|c|c|c|c|}
\hline & \multicolumn{3}{|c|}{ Day 0 } & \multicolumn{3}{|c|}{ Day +7 } \\
\cline { 2 - 7 } & $\begin{array}{c}\text { TB } \\
(\mathrm{n}=6)\end{array}$ & $\begin{array}{c}\text { NTB } \\
(\mathrm{n}=9)\end{array}$ & $\begin{array}{c}\text { PF } \\
(\mathrm{n}=5)\end{array}$ & $\begin{array}{c}\text { TB-r } \\
(\mathrm{n}=5)\end{array}$ & $\begin{array}{c}\text { NTB } \\
(\mathrm{n}=9)\end{array}$ & $\begin{array}{c}\text { PF } \\
(\mathrm{n}=5)\end{array}$ \\
\hline 5-HT & $2,3 \pm 0,2^{*+}$ & $1,5 \pm 0,1$ & $1,7 \pm 0,3$ & $1,1 \pm 0,1^{*}+\mathrm{o}$ & $1,5 \pm 0,1$ & $0,4 \pm 0,0^{*} \mathrm{o}$ \\
\hline 5-HIAA & $392 \pm 49^{*+}$ & $244 \pm 23$ & $100 \pm 17^{*}$ & $99 \pm 27^{*+o}$ & $244 \pm 23$ & $34 \pm 3^{* \mathrm{o}}$ \\
\hline DA & $8,0 \pm 0,6^{*+}$ & $14,2 \pm 1,0$ & $10,5 \pm 0,7^{*}$ & $8,5 \pm 0,7^{*+}$ & $14,2 \pm 1,0$ & $11,3 \pm 0,7$ \\
\hline DOPAC & $42,3 \pm 5,6^{*+}$ & $30,2 \pm 2,0$ & $10,2 \pm 0,9^{*}$ & $16,5 \pm 1,5^{*+\mathrm{o}}$ & $30,2 \pm 2,0$ & $7,6 \pm 1,4^{*}$ \\
\hline
\end{tabular}

VMN-DA was significantly lower both in PF and TB rats vs NTB group $(\mathrm{p}<0.01)$ during anorexia on Day 0 . This reduction was more dramatic in TB vs PF animals $(\mathrm{p}<$ $0.01)$. In spite of the similar pattern of VMN-DA in both TB and PF rats, DOPAC was higher in the TB $(\mathrm{p}<0.01)$ and lower in the PF $(\mathrm{p}<0.01)$ vs NTB rats.

Seven days after the TB-r rats had tumor resected on Day +7, both the VMN-5-HT and 5-HIAA in TB-r group had decreased and reached even lower levels significantly vs NTB controls $(p<0.01)$. The depressed 5-HT and 5-HIAA were measured in the PF group, being significantly lower vs NTB $(\mathrm{p}<0.01)$ and TB-r $(\mathrm{p}<0.01)$.

As during anorexia, also after tumor removal on Day +7 the VMN-DA remained significantly lower in TB-r vs NTB group ( $\mathrm{p}<0.01$ ). The lower VMN-DA in PF rats on Day +7 was not significantly different vs NTB controls. On the contrary, VMN-DOPAC, which was significantly higher during anorexia ( $p<0.01$ vs NTB), had significantly decreased after tumor removal in TB-r vs NTB control $(p<0.01)$, while in the PF animals DOPAC remained significantly depressed both during anorexia on Day 0 ( $\mathrm{p}<0.01$ vs NTB) and after tumor removal on Day +7 ( $p<0.01$ vs NTB).

Eating-Related VMN-Monoamines Changes. The eatingrelated changes in VMN-5-HT, 5-HIAA, DA and DOPAC as expressed in the percentage of baseline, are shown in Fig.s 4 and 5.

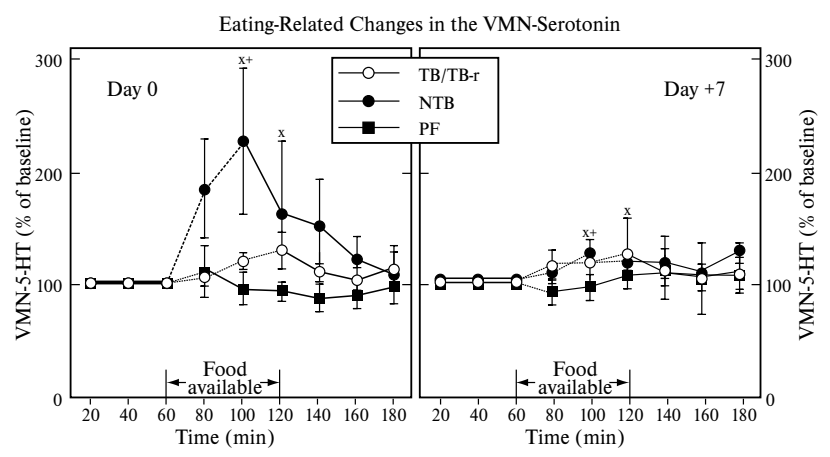

Fig. 4A: Eating-related VMN-5-HT changes during anorexia on Day 0 (left) and after tumor removal on Day +7 (right), as expressed in the percentage of baseline. The results are shown as mean $\pm \mathrm{SE}$ for tumor-bearing $(\mathrm{TB}, \mathrm{n}=6)$, $\mathrm{TB}$ after tumor removal (TB-r, $n=5$ ), non-tumor-bearing controls $(\mathrm{NTB}, \mathrm{n}=9)$ and non-tumor-bearing pair-fed $(\mathrm{PF}, \mathrm{n}=5)$ rats. Food was available between 60 and $120 \mathrm{~min}$. Broken line indicate duration of actually eating - note this varies between groups; the solid line indicate period while not eating. Sampling was done every 20 min. Significance ${ }^{x} p<0.05$ in NTB, ${ }^{+} \mathrm{p}<0.05$ in TB/TB-r vs baseline.

Eating was associated with VMN-5-HT increase in all groups, but the respective rises had different pattern. In the TB group, 5 -HT reached its zenith $(230 \%)$ during the meal 
at $20-40 \mathrm{~min}$ ( $\mathrm{p}<0.05$ vs baseline) being significantly higher vs NTB ( $p<0.05)$, and after that gradually returned to the premeal level, while 5-HT in NTB rats showed a more delayed increase, reaching its zenith (134\%) during the 40 60 min interval ( $p<0.05$ vs baseline) and only then did it begin to decrease towards the baseline level. After the TB rat had their tumor removed, 5-HT peaked at $127 \%$ during 20-40 min samples ( $p<0.05$ vs baseline) and in NTB at $124 \%$ at $40-60 \min (\mathrm{p}<0.05$ vs baseline), and there was not any significant difference between TB-r and NTB rats (Fig. 4A).

The VMN-5-HIAA started to decrease with eating in all groups, and was significantly lower also post-eating in TB and NTB animals ( $p<0.05$ vs baseline) (Fig. 4B).

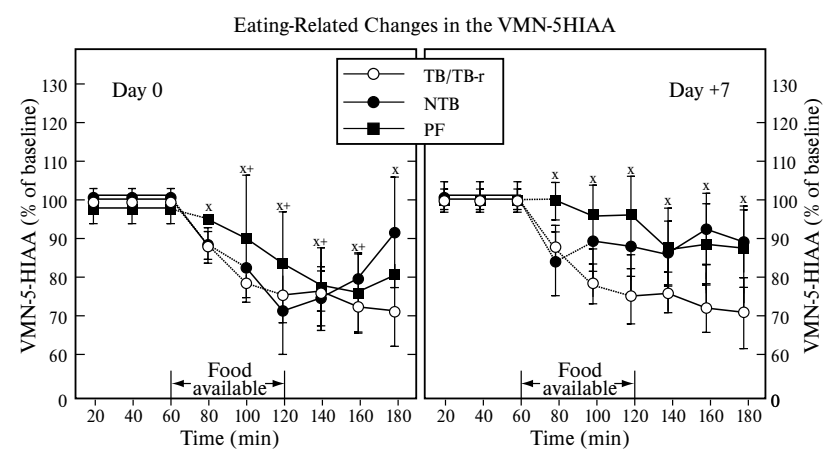

Fig. 4B: Eating-related VMN-5-HIAA changes during anorexia on Day 0 (left) and after tumor removal on Day +7 (right), as expressed in the percentage of baseline. The results are shown as mean $\pm \mathrm{SE}$ for tumor-bearing $(\mathrm{TB}, \mathrm{n}=6)$, TB after tumor removal (TB-r, $n=5$ ), non-tumor-bearing controls (NTB, n=9) and non-tumor-bearing pair-fed (PF, $\mathrm{n}=5$ ) rats. Food was available between 60 and $120 \mathrm{~min}$. Broken line indicate duration of actually eating - note this varies between groups; the solid line indicate period while not eating. Sampling was done every $20 \mathrm{~min}$. Significance $\mathrm{x}_{\mathrm{p}}<0.05$ in NTB, ${ }^{+} \mathrm{p}<0.05$ in TB/TB-r vs baseline.

Despite the similar pattern, the changes in TB-r and PF rats were not significant.

VMN-DA showed almost a mirror image from 5-HT during eating in TB, TB-r, PF and in NTB animals, as DA was decreasing with eating ( $p<0.05$ vs baseline). After eating, DA in TB rats returned to the preeating values, while in NTB rats DA was still depressed ( $p<0.05$ vs baseline). In the PF rats, the meal produced a similar response as in NTB during the first microdialysis (e.g. DA decreased, $\mathrm{p}<$ 0.05 vs baseline). With prolonged malnutrition of PF rats, eating also decreased VMN-DA seven days later, but without statistical significance (Fig. 5A).

VMN-DOPAC regularly decreased during and after eating in non-anorexic NTB and PF rats ( $p<0.05$ vs baseline), but no significant changes occured in the anorexic TB group, as DOPAC remained at the baseline level both during and af- ter eating. Eating was associated with decreased DOPAC which was not significant in TB-r rats or in malnourished PF rats during their second microdialysis at Day +7 (Fig. 5B).

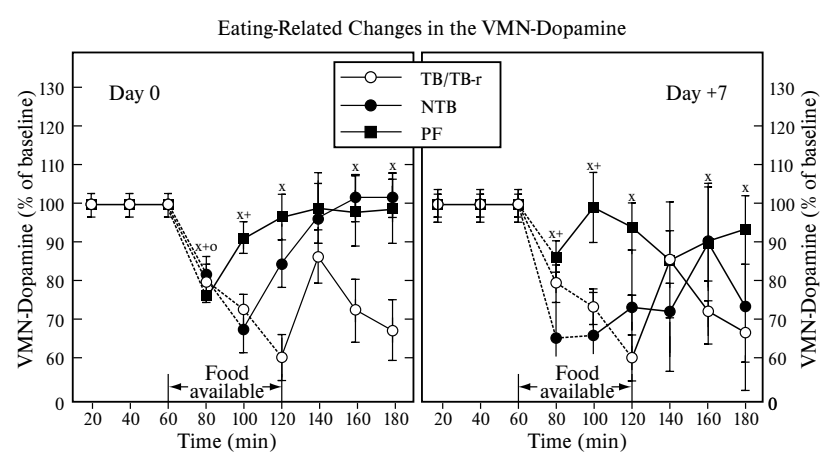

Fig. 5A: Eating-related VMN-DA changes during anorexia on Day 0 (left) and after tumor removal on Day +7 (right), as expressed in the percentage of baseline. The results are shown as mean \pm SE for tumor-bearing $(T B, n=6)$, TB after tumor removal (TB-r, $\mathrm{n}=5$ ), non-tumor-bearing controls $(\mathrm{NTB}, \mathrm{n}=9)$ and non-tumor-bearing pair-fed $(\mathrm{PF}, \mathrm{n}=5)$ rats. Food was available between 60 and 120 min. Broken line indicate duration of actually eating - note this varies between groups; the solid line indicate period while not eating. Sampling was done every $20 \mathrm{~min}$. Significance ${ }^{+} \mathrm{p}<0.05$ in TB/TB-r , ${ }^{\mathrm{x}} \mathrm{p}<0.05$ in NTB, ${ }^{\circ} \mathrm{p}<0.05$ in PF vs baseline.

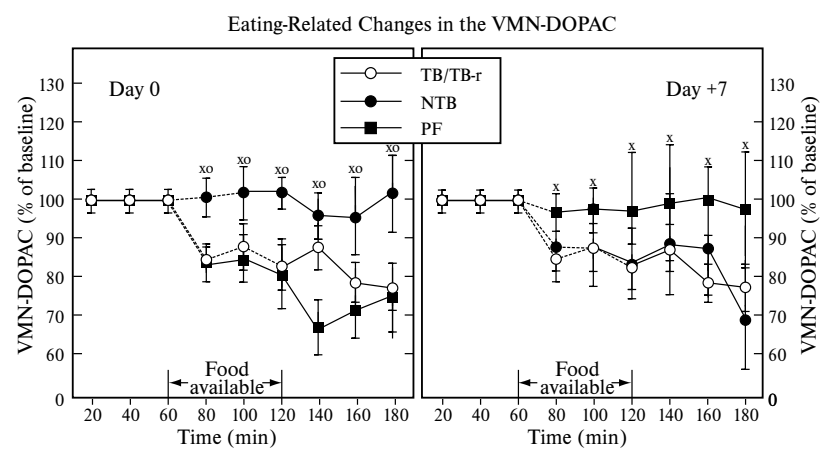

Fig. 5B: Eating-related VMN-DOPAC changes during anorexia on Day 0 (left) and after tumor removal on Day +7 (right), as expressed in the percentage of baseline. The results are shown as mean \pm SE for tumor-bearing $(T B, n=6)$, TB after tumor removal (TB-r, $n=5$ ), non-tumor-bearing controls $(\mathrm{NTB}, \mathrm{n}=9)$ and non-tumor-bearing pair-fed $(\mathrm{PF}, \mathrm{n}=5)$ rats. Food was available between 60 and $120 \mathrm{~min}$. Broken line indicate duration of actually eating - note this varies between groups; the solid line indicate period while not eating. Sampling was done every $20 \mathrm{~min}$. Significance ${ }^{+} \mathrm{p}<0.05$ in TB/TB-r, ${ }^{\mathrm{x}}<0.05$ in NTB, ${ }^{o} \mathrm{p}<0.05$ in PF vs baseline.

\section{Discussion}

Baseline and eating-related dynamic changes of the VMN serotonergic and dopaminergic activities were mea- 
sured and compared among anorectic tumor-bearing, control and pair-fed rats using in vivo brain microdialysis. The results provide direct evidence that the VMN-monoamine neurotransmitters are involved in cancer-induced anorexia:

1) Higher release of VMN 5-HT and lower release of DA, with increased metabolism of both neurotransmitters as indicated by higher 5-HIAA and DOPAC, were found in anorectic TB vs NTB rats at the baseline;

2) While the food intake in TB rats was significantly lower, they released significantly more of VMN-5-HT during eating. Onset of satiation was concomitant with a decreasing VMN-DA, while its catabolite DOPAC did not change. 3) Enhanced serotonergic neuronal activity, and uncoupled dopaminergic release/metabolism were related to cancer anorexia and did not result from malnutrition per se; thus a divergent pattern with lower VMN 5-HT and 5-HIAA, and/or higher DA and lower DOPAC was measured in pair-fed vs TB animals. 4) Seven days after tumor removal in the tumor-bearing animals, VMN 5-HT, 5-HIAA and DOPAC decreased, while VMN-DA did not change significantly and remained low. The daily food intake was nearly normal seven days after tumor removal, but was still low during microdialysis, probably reflecting the low VMN-DA release and turnover.

5-HT in the medial hypothalamus is classically recognized as an eating-inhibitory neurotransmitter in the normal rat. Numerous pharmacological manipulations using precursors, agonists, or transmission-enhancers all have contributed evidence in support $(2,16,28,33,35)$. Anorectic drugs, such as mefenorex, proceed mainly by means of these serotonergic properties (30). Consequently, increased brain 5-HT metabolism was observed in comprehensive studies of Fischer and Chance in anorectic tumor-bearing rats, analysing brain tissue homogenates $(5,7,8,37)$. When cancer anorexia was defined as in our study, e.g., mean food intake by MCA-bearing rats was at least $1-1.5 \mathrm{~g} / 100 \mathrm{~g}$ body weight less than the nontumor-bearing group for 3 consecutive days, increased hypothalamic 5-HT and 5-HIAA were consistently reported $(7,9)$. However, these changes were located only extrahypothalamically, when more stringent criterion for MCA-induced cancer anorexia was used, namely food intake less than $3 \mathrm{~g} / 100 \mathrm{~g}$ body weight for 2 consecutive days (5). Regional changes in brain indole concentrations of anorectic MCA-bearing male Fischer 344 rats were more widespread than in the female SpragueDawley rats bearing Walker 256 carcinosarcoma (a more acute model of cancer anorexia) (5). Apparently, some aspects of regional hypothalamic monoamine changes are dependent on the tumor type and size, and furthermore, could be easily obscured in the whole hypothalamic assays.

From microdialysis studies it was proposed, that the involvement of brain monoamines in the control of food intake may have expressed the endocrine and metabolic events that accompany a meal, rather than the motivational state of the animal itself, eg. hunger, satiation and satiety in the NTB rats (29). A complex cascade of peripheral events that affect hypothalamic appetite-regulating mechanisms via humoral factors (26), produced either by the neoplastic tissue or by the host in response to the tumor, is evidenced in cancer (25). Our results are in agreement with this concept, because both VMN-serotonergic and dopaminergic systems were affected in cancer anorexia and experimentally-induced hunger in the pair-fed animals. Nevertheless, the pattern was different in the particular groups, indicating cancer-specific involvement of VMN monoamine systems in mediating the anorectic status. In TB rats, VMN-5-HT rose significantly during eating and fell to the baseline afterwards. It is noteworthy that in spite of its allready significantly higher baseline, 5-HT release increased by $230 \%$ of baseline in TB rats and only by $130 \%$ of baseline in NTB rats. This might be a consequence of the increased availability of tryptophan as 5-HT precursor in the plasma (19) and in the hypothalamus $(5,7,37)$ of anorectic TB rats. The increase of 5-HT in the ventromedial nucleus of hypothalamus was directly related to the tumor, because:

1) baseline VMN-5-HT was not more increased seven days after tumor removal;

2) after tumor resection, VMN-5-HT release during eating was not quantitatively different vs NTB rats;

3) VMN-5-HIAA was not more increased seven days after tumor removal; and

4) VMN-5-HT and 5-HIAA were not increased but decreased in the pair-fed rats, which were nutritionally depleted. Since 5-HT metabolism is precursor-driven, this is further support that serotonergic metabolism is not increased due to the malnutrition but to the anorexia.

We extended the previously observed hypothalamic monoamine changes during cancer anorexia by performing microdialysis in vivo and finding decreased baseline VMNDA and increased DOPAC in anorectic TB rats. The function of DA in the central control of food intake is not clearly understood, but varies within different hypothalamic areas, and can be involved in at least several functions: locomotion, feeding, behavior reinforcement and central reward mechanisms (4). In vivo, the descending slope of DA has been considered to cause the satiation that stops feeding (28). Since the medial hypothalamic area has been shown rather refractory to DA injections in regard to food intake (14), one may not expect a feeding inhibitory role of VMN-DA, when it increases. An enhanced breakdown with depressed release of DA in TB rats, as seen in the present study, is thus presumed to trigger the status of chronic satiety, while a hunger sensation in food-restricted PF rats is associated with depressed DA turnover. Because VMN-5-HT was not more increased after tumor removal, we propose that the disturbances in DA release and metabolism may be associated with non-normalization of food intake while microdialysing the VMN of TB-r animals. Our microdialysis data, obtained during spontaneous eating, support the finding that a rise in VMN-DA does not have an inhibitory effect on feeding. Instead, a descending slope of VMN-DA may be involved in removal of the satiety status. When ea- 
ting-related changes in rostromedial dopaminergic monoamines and their respective metabolites were examined during transitions between hunger and satiety, induced by infusing insulin with glucose or infusing insulin with food available, DA decreased similarily as with eating in our present study (29). However, in contrast to the present study, DA synthesis and release were dissociated, indicating different metabolic turnover in the particular rat model used.

Recent investigation has addressed several links between neurotransmitters and other factors which modulate food intake in cancer anorexia. Neuropeptide Y (NPY), a potent orexigenic agent in the rat hypothalamus (36), is less effective in TB rats (11), and hypothalamic concentration of NPY is decreased in TB rats (10). On the other hand, DA release is typically enhanced by NPY essentially in all structures of cerebral tissue (3). We propose that the depressed hypothalamic NPY activity may be associated with decreased DA release in anorectic TB rats. Cytokines are among other candidates which may influence release and metabolism of neurotransmitters. For example, interleukin-1b increases hypothalamic serotonergic neurotransmission (22) and augments release of norepinephrine, DA and 5-HT in the rat hypothalamus (34). Therefore it is likely that tumor-induced aberations of neurochemical mechanisms which result in cancer anorexia are quite complex. Our current efforts are focused on the search of mechanisms linking the metabolic changes during tumor growth with the neurotransmitters which are critically involved in feeding behavior.

\section{References}

1. Blaha V, Yang Z-J, Meguid MM, Laviano A, Zadak Z, Rossi Fanelli F. Cancer anorexia is modulated by interaction of hypothalamic-VMN dopamine (DA) \& serotonin (5HT) \& and not solely 5-HT as currently thought. Surg Forum 1996;47:517-20.

2. Blundell JE. Serotonin and appetite. Neuropharmacol 1984;23:1537-52.

3. Heilig M, Vecsei L, Wahlestedt C, Alling C, Widerlov E. Effects of centrally administered neuropeptide Y (NPY) and NPY 13-36 on the brain monoaminergic systems of the rat. J Neural Transm 1990;79:193-208.

4. Hoebel BG, Hernandez L, Schwartz DH, Mark GP, Hynter GA. Microdialysis studies of brain norepinephrine, serotonin, and dopamine release during ingestive behavior: Theoretical and clinical implications. Ann NY Acad Sci 1989;575:171-93.

5. Chance WT, von Meyenfeldt MF, Fischer JE. Changes in brain amines associated with cancer anorexia. Neurosci. Biobehav Rev 1983;7:471.

6. Chance WT, von Meyenfeldt MF, Fischer JE. Serotonin depletion by 5,7-dihydroxytryptamine or para-chloroamphetamine does not affect cancer anorexia. Pharmacol Biochem Behav 1983;18:115.

7. Chance WT, Cao L, Nelson JL, Foley-Nelson T, Fischer
JE. Reversal of neurochemical aberrations after tumor resection. Am J Surg 1988;155:124-30.

8. Chance WT, Zhang FS, Foley-Nelson T, Fischer JE. Hyperammonemia and anorexia in Morris Hepatoma-bearing rats. Physiol Behav 1991;50:397-401.

9. Chance WT, Cao L, Fischer JE. Tumor-induced alterations in brain neurotransmitter and plasma ammonia concentrations are normalized twenty-four hours after tumor resection. Life Sci 1991;48:425-32.

10. Chance WT, Balasubramaniam A, Dayal R, Brown J, Fischer J E. Hypothalamic concentration and release of neuropeptide $\mathrm{Y}$ into microdialysates is reduced in anorectic tumor-bearing rats. Life Sci 1994;54:1869-74.

11. Chance WT, Balasubramaniam A, Fischer JE. Neuropeptide Y and the development of cancer anorexia. Ann Surg 1995;221:579-89.

12. Kurzer M, Janiszewski FJ, Meguid MM. Amino acid profiles in tumor-bearing and pair-fed non tumor-bearing malnourished rats. Cancer 1988;62:1492-6.

13. Laviano A, Meguid MM, Renvyle T, Opara EI, Yang Z.J, Rossi Fanelli F. Il-1a acts directly on hypothalamic VMH to mediate cancer anorexia. Surg Forum 1995;46:491-3.

14. Leibowitz SF, Rossakis C. Pharmacological characterization of perifornical hypothalamic dopamine receptors mediating feeding inhibition in the rat. Brain Res 1979;172: 115-30.

15. Leibowitz SF. Neurochemical systems of the hypothalamus. Control of feeding and drinking behaviour and waterelectrolyte excretion. In: Morgane PJ, Panksepp J, eds. Handbook of the hypothalamus. New York: Marcel Dekker, 1980:299-437.

16. Leibowitz SF. Brain monoamines and peptides: role in the control of eating behavior. Federatio Proc 1986;45: 1396-1403.

17. Madden RE, Burk D. Production of viable single cell suspension from solid tumors. J Natl Cancer Inst 1961;27: 841-61.

18. McCarthy HD, McKibbin PE, Perkins AV, Linton EA, Williams G. Alterations in hypothalamic NPY and CRF in anorexic tumor-bearing rats. Am J Physiol 1993;264: E638E643.

19. Meguid MM, Yang Z-J, Koseki M. Eating-induced rise in LHA-dopamine correlates with meal size in normal and bulbectomized rats. Brain Res Bull 1995;36:487-90.

20. Meguid MM, Yang Z-J, Gleason JR. The gut-brain brain-gut axis in anorexia: toward an understanding of food intake regulation. Nutrition 1996;12:S57-S62.

21. Meguid MM, Yang Z-J, Laviano A. Meal size and meal number: relationship to dopamine levels in the ventromedial hypothalamic nucleus. Submitted to Am J Physiol, 1996. 22. Mohankumar PS, Thyagarajan S, Quadri SK. Interleukin-1b increases 5-hydroxyindolacetic acid release in the hypothalamus in vivo. Brain Res Bull 1993;31: 745-8. 23. Morrison SD. Contribution of reduced hunger and premature satiety to cancerous hypophagia in rats. Cancer Res 1984;44:1041-3. 
24. Muscaritoli M, Meguid MM, Beverly JL, Yang Z-J, Cangiano C, Rossi-Fanelli F. Mechanism of early tumor anorexia. J Surg Res 1996;60:389-97.

25. Nelson KA, Walsh D, Sheehan FA. The cancer anorexia-cachexia syndrome. J Clin Oncol 1994;12:213-25.

26. Norton JA, Moley JF, Green MV, Carson RE, Morrison SD. Parabiotic transfer of cancer anorexia/cachexia in male rats. Cancer Res 1985;45:5547-9.

27. Opara EI, Laviano A, Meguid MM. Correlation between food intake and CSC IL-1a in anorectic tumor bearing rats. NeuroReport 1996;6:750.

28. Orosco M, Nicolaidis S. Spontaneous feeding-related monoaminergic changes in the rostromedial hypothalamus revealed by microdialysis. Physiol Behavior 1992;52: 10154-1019. Orosco M, Nicolaidis S. Insulin and glucose-induced changes in feeding and medial hypothalamic monaomines revealed by microdialysis in rats. Brain Res Bull 1994;33: 289-97.

29. Orosco M, Moret C, Briley M, Nicolaidis S. Effect of Mefenorex on 5-HT release: studies in vitro on rat hypothalamic slices and in vivo by microdialysis. Pharmacol Biochem Behav 1995;50:485-90.

30. Parada MA, Hernandez L, Puig De Parada M, Paez X, Hoebel BG. Dopamine in the lateral hypothalamus may be nvolved in the inhibiton of locomotion related to food and water seeking. Brain Res Bull 1990;25:961.

31. Pellegrino LJ, Pellegrino AS, Cushman AJ. A stereotaxic atlas of the rat brain. 2nd ed. New York: Plenum Press, 1986.

32. Samanin R, Mennini T, Garattini S. Evidence that it is possible to cause anorexia by increasing release and/or directly stimulating postsynaptic serotonin receptors in the brain. Prog Neuropsychopharmacol 1980;4:363-9.
33. Shintani F, Nakaki T, Kanba $\mathrm{S}$ et al. Interleukin-1b augments release of norepinephrine, dopamine, and serotonin in the rat hypothalamus. J Neurosci 1993;13:3574-81.

34. Schwartz DH, Hernandez L, Hoebel BG. Serotonin release in lateral and medial hypothalamus during feeding and its anticipation. Brain Res Bull 1990;25:797-802.

35. Stanley BG, Leibowitz SF. Neuropeptide Y injected into the paraventricular hypothalamus: a powerful stimulant of feeding behavior. Proc Natl Acad Sci USA 1985;82:3940.

36. Von Meyenfeldt M, Chance WT, Fischer JE. Correlation of changes in brain indoleamine metabolism with onset of anorexia in rats. Am J Surg 1982;143:133.

37. Yang Z-J, Meguid MM. Continuous systemic interleukin-1a infusion suppresses food intake without increasing lateral hypothalamic dopamine activity. Brain Res Bull 1995;36:417-20.

38. Yang Z-J, Koseki M, Meguid MM. Laviano A. Eatingrelated increase of dopamine concentration in the LHA associated with oronasal stimulation. Am J Physiol 1996;270: R315-R318.

Submitted December 1997.

Accepted January 1998.

MUDr. Vladimír Bláha, CSc., Dept. of Metabolic Care and Gerontology, Faculty of Medicine and Teaching Hospital, 50005 Hradec Králové, Czech Republic 